The Principles of Probability and Approximations in Arithmetic

Author(s): W. Hope-Jones

Source: The Mathematical Gazette, Vol. 9, No. 127 (Jan., 1917), pp. 5-8

Published by: Mathematical Association

Stable URL: http://www.jstor.org/stable/3602973

Accessed: 17-10-2015 04:25 UTC

Your use of the JSTOR archive indicates your acceptance of the Terms \& Conditions of Use, available at http://www.jstor.org/page/ info/about/policies/terms.jsp

JSTOR is a not-for-profit service that helps scholars, researchers, and students discover, use, and build upon a wide range of content in a trusted digital archive. We use information technology and tools to increase productivity and facilitate new forms of scholarship. For more information about JSTOR, please contact support@jstor.org. 
Note.-Since writing this paper I have read those portions of Heaviside's Electromagnetic Theory dealing with vectorial algebra and analysis, and giving incidentally* some idea of the controversy toward the close of the last century for and against the use of quaternions in mathematical physics. I am very glad that before beginning the study of vector analysis I had no knowledge of quaternions beyond the name and a few vague ideas about the controversy referred to. I was thus able to approach the subject quite unbiassed and from the Cartesian point of view. This, I believe, makes all the difference to one's reception of the vectorial method; for, as stated at the close of my paper, the Cartesian and vectorial analyses are inseparably connected. Since writing those lines, I was pleased to find the same thought expressed by Heaviside. "The quaternionists," he says, "want to throw away the 'Cartesian trammels,' as they call them. This may do for quaternions, but with vectors would be a grave mistake. My system, so far from being inimical to the Cartesian system of mathematics, is its very essence." $\dagger$

The unfortunate idea that vector analysis is a sort of modified system of quaternions is perhaps largely responsible for its tardy adoption in Great Britain. The consideration of my own experience may therefore be of some value, chiefly because there were no initial conceptions and associations to bias me. The first book I studied on the subject was the French edition of Le Calcul Vectoriel, by Burali-Forti and Marcolongo. I found their system interesting and helpful, though not always natural. While waiting for other books by mail I read Silberstein's Vectorial Mechanics, in which Heaviside's notation and analysis are employed. Speaking only of the chapter on vector algebra and analysis, I thought it simple and direct, but found the symbol $V$ used to denote a vector product very confusing. I have since, of course, learnt that this is only a survival of the quaternionic notation, and yet adopted, strange to say, by such a pronounced anti-quaternionist as Heaviside. Then I had the pleasure of reading Gibb's Vector Analysis, by E. B. Wilson, explaining a system substantially the same as that of Heaviside, from which it differs chiefly in notation. It appeared to me at once most natural in both method and notation, and afforded me the inspiration already referred to. The bracket notation for products of vectors has since come under my notice in many works, but while reading this with equal facility I prefer that of Gibbs. Not only does this leave the brackets free for ordinary algebraic purposes, but the dot and cross are now hardly needed in algebra to indicate a product.

23rd September, 1916,

Ormond College, Parkville, Melbourne.

\section{THE PRINCIPLES OF PROBABILITY AND APPROXIMATIONS IN ARITHMETIC.}

\section{$\mathrm{Br}$ W. Hope-Jones, B.A.}

I SUPPose that most teachers of Elementary Mathematics, when first they broach the subject of finding the circumference of a circle, write on their blackboards for comparison $\quad \pi=3 \cdot 14159$,

$$
3 \frac{1}{1}=3 \cdot 14286
$$

* In a manner often blunt and amusing, but always interesting.

† Loc. cit., vol. i. p. 305. 
and draw the conclusion that "as these results agree as far as the third figure, the approximation $\pi=3 \frac{1}{7}$ can reasonably be expected to get the first 3 figures of your answer right." The purpose of this paper is to analyse to some extent a few practical rules of this type.

1. A diameter, chosen at random, and reckoned in any units, is multiplied by $3 \frac{1}{7}$, and the result given correct to 3 significant figures. Find the probability that the circumference so obtained is correct to 3 significant figures.

Let the exact circumference, multiplied or divided by some integral power of 10 , be $c$ units, $c$ being between 100 and 1000 . Then the approximate circumference, similarly multiplied or divided, $=\frac{3 \frac{1}{7} c}{\pi}$.

The very rare case in which $c<1000$ but $\frac{3 \frac{1}{\pi} c}{\pi}>1000$ is left out of account, and terms involving powers of $\left(3 \frac{1}{7}-\pi\right)$ are omitted. The frequency of $c$ is not uniform, but can be proved inversely proportional to $c$.

Proof. If $x$ is a circumference "chosen at random," let the probability that $x$ is between $X$ and $X+d X$ be $f(X) d X$.

Then the probability that $b x$ is between $b X$ and $b X+b d X=f(X) d X$, $b$ being any constant.

But this probability is by definition $f(b X) d(b X), b X$ being also "chosen at random."

$$
\therefore f(X) d X=f(b X) d(b X), \frac{f(b X)}{f(X)}=\frac{1}{b} \text { and } f(X) \propto \frac{1}{X} \text {. }
$$

That is, the frequency of $X$ is proportional to $\frac{1}{X}$, so that the frequency of $c=\frac{B}{c}, B$ being constant. The probability of a value between $X$ and $X+d X=\frac{B d X}{X}=B d(\log X)$, so that all values of $\log X$ are equally likely. Values of $c$, in fact, are distributed uniformly along a slide-rule, not along a tape-measure.

In practice it is impossible to find quantities which are chosen perfectly at randon : but a rough experimental verification of this result can be found in facts of the following kind. Of the 364 towns and London boroughs mentioned in the Municipal Directory of England and Wales in Whitaker's Almanack for 1914 , the populations of 108 begin with the figure 1 ; and $\frac{108}{364}(=0.297)$ is a good approximation to $\frac{\log 2}{\log 10}$, that is $\frac{\int_{1}^{2} \frac{B d X}{X}}{\int_{1}^{10} \frac{B d X}{X}}$.

The correctness of the corresponding approximate circumference depends on whether or not an integer $+\frac{1}{2}$ comes between $c$ and $\frac{3 \frac{1}{4} c}{\pi}$. Hence, for the approximate circumference to be correct, it is necessary that $c$ should not lie within certain "prohibited regions," namely, from $\frac{\left(n+\frac{1}{2}\right) \pi}{37}$ to $\left(n+\frac{1}{2}\right)$.
$(n=100,101, \ldots 999$.

The width of the "prohibited region" is proportional to $n+\frac{1}{2}$, and so approximately to $c$. Strictly speaking, we cannot say that the probability of $c$ falling within a "prohibited region" is proportional to the magnitude of $c$, because for any given value of $c$ this probability is either exactly 0 or exactly 1 : but, taking the à priori probability of $c$ falling into a "prohibited region" in any particular neighbourhood as the fraction which "prohibited regions" form of the whole range of $c$ in that neighbourhood, we may say that the probability of an integer $+\frac{1}{2}$ coming between $c$ and $\frac{3 \frac{1}{4} c}{\pi}$ is $\frac{3 \frac{1}{7} c}{\pi}-c$,
that is, the width of the "prohibited region." 
Let the whole probability of the approximate circumference being incorrect to 3 figures be $A$;

$$
\begin{aligned}
\therefore A & \left.=\int_{100}^{1000}\left(\frac{3 t_{c} c}{\pi}-c\right)(\text { frequency of } c) d c / \int_{100}^{1000} \text { (frequency of } c\right) d c \\
& =\int_{100}^{1000}\left(\frac{3 \frac{1}{7} c}{\pi}-c\right) \frac{B d c}{c} / \int_{100}^{1000} \frac{B d c}{c} \\
& =900\left(\frac{3 \frac{1}{7}}{\pi}-1\right) /\left(\log _{e} 1000-\log _{e} 100\right)=900\left(\frac{3 \frac{1}{7}}{\pi}-1\right) \log _{10} e=\cdot 15732 \ldots
\end{aligned}
$$

$\therefore$ the common rule that "the approximation $\pi=3 \frac{1}{7}$ will generally get the first 3 figures of your answer right" is a sound working rule, because it will succeed in 843 cases out of 1000 , chosen at random, supposing the answer to contain $\pi$ as a factor. The same applies to $\frac{1}{\pi}$; and if the factor is $\pi^{ \pm \frac{1}{2}}$, the chance of error will naturally be reduced from $\cdot 157$ to 079 .

The result (1) can be generalised as follows:

If, in a quantity containing $p$ as a factor, $P$ be substituted as an approximation for $p, \frac{P \sim p}{p}$ being less than $10^{-n}$, the probability that the answer is incorrect to $n$ significant figures is

$$
9.10^{n-1}\left(\frac{p}{p} \sim 1\right) \log _{10} \text { e, i.e. } 3 \cdot 909\left(\frac{p}{p} \sim 1\right) 10^{n-1} \text {. }
$$

Another important special case of this is the approximation, 1 cubic foot $=6 \frac{1}{4}$ gallons.

Here $P=6 \cdot 25, p=6.23211$ ( $v$. Chambers's Mathematical Tables), and $n=$ any integer less than 3 . ( $n=2$ gives the most useful result.) Then the probability that a quantity, given accurately in cubic feet, will be reduced to gallons, correct to 2 figures, by the $6 \frac{1}{4}$ approximation, is

$$
1-3 \cdot 909\left(\frac{6 \cdot 25}{6 \cdot 23211}-1\right) \times 10=1-\cdot 112=\cdot 888 \text {. }
$$

By a sinilar substitution we find that if a number of miles is reduced to $\mathrm{Km}$., by taking 5 miles $=8 \mathrm{Km}$., the result will be correct to 2 figures in 772 casés out of 1000 .

2. Returning to the approximation $\pi=3 \frac{1}{\pi}$, it is evident that it will sometimes give an answer correct to 4 figures. Let us find how often this will happen.

$$
\text { In the result (1), } A=\int_{100}^{1000}\left(\frac{3 \frac{1}{7} c}{\pi}-c\right) B \frac{d c}{c} / \int_{100}^{1000} B \frac{d o}{c}
$$

the effect of dealing with the 4 th instead of the 3rd figure will be to alter the lower limit in both integrals to 1000 , and the upper limit in the de. nominator to 10,000 : but in the numerator, before $c$ reaches 10,000 , the width of the "prohibited regions" $\left(\frac{3 \frac{1}{7} c}{\pi}-c\right)$ will have increased to 1 , so that above that limit $c$ cannot possibly keep out of them, and the approximate answer is bound to be wrong. This begins to occur as soon as $\frac{3 \frac{1}{7} c}{\pi}-c$ rises to 1 , that is when $c=\frac{\pi}{3 \frac{1}{7}-\pi}$. 
$\therefore$ the chance that the approximation $\pi=3 \frac{1}{7}$ will give an answer incorrect to 4 figures is

$$
\begin{aligned}
& \left\{\int_{1000}^{\frac{\pi}{3 !-\pi}}\left(\frac{3 \frac{1}{7} c}{\pi}-c\right) B \frac{d c}{c}+\int_{\frac{\pi}{3 !-\pi}}^{10000} B \frac{d c}{c}\right\} / \int_{1000}^{10000} B \frac{d c}{c} \\
& =\left\{\left(\frac{3 \frac{1}{7}}{\pi}-1\right)\left(\frac{\pi}{3 \frac{1}{7}-\pi}-1000\right)+\log _{e} 10000-\log _{e}\left(\frac{\pi}{3 \frac{1}{7}-\pi}\right)\right\} / \log _{e} 10 \\
& =\left\{1-1000\left(\frac{3 \frac{1}{4}}{\pi}-1\right)\right\} \log _{10} e+4+\log _{10}\left(\frac{3 \frac{1}{7}-\pi}{\pi}\right)=\cdot 864 \text {. }
\end{aligned}
$$

This gives us the complete answer to the question, "Why should I give the answers to circle questions correct to 3 figures and not to 4 ?" "Because if you try for 3 figures, you will get them right more than 5 times out of $6:$ but if you try for a 4 th figure, it will be wrong more than 6 times out of 7."

The result (3) may be generalised thus:

Defining $p$ and $P$ as before, $\frac{P \sim p}{p}$ being between $10^{-n}$ and $10^{-n-1}$, the probability that the approximate answer is incorrect, if given to $n+1$ figures, is

$$
\left\{1-10^{n}\left(\frac{P}{p} \sim 1\right)\right\} \log _{10} e+(n+1)+\log _{10}\left(\frac{P}{p} \sim 1\right) .
$$

The minimum value of this being 3909 , the $(n+1)$ st figure is not, in general, trustworthy.

The following table is of value in deciding how many figures we should give up, or teach our pupils to give up, in answers obtained by using certain common approximations :

\begin{tabular}{l|ccc}
\hline \multicolumn{1}{|c|}{ Approximation. } & \multicolumn{3}{|c}{ Probability of obtaining answer correct to } \\
significant figures.
\end{tabular}

( $T_{0}$ be continued.)

\section{MATHEMATICAL NOTES.}

500. [X. iv. a.] Required, a Solution of the following :

$E W$ is a straight line, $P_{p}$ is parallel to $E W$ at a distance $A ; Q q$ is parallel to $P p$ at a distance $B ; R r$ is parallel to $Q q$ at a distance $C$. $P, Q, R$ are any points on $P p, Q q, R r$.

$E W$ represents a road running $E$ and $W$. The three strips, which we may call $A, B, C$, are strips of cultivated land, the speeds across which are respectively $1 / a, 1 / b$, and $1 / c$ of the speed on the road. Speeds equal to that on the road are possible along the three parallel tracks $P p, Q q, R r$.

At a given point on $E W$ an order is received to reach the position $P$ in the shortest time. What is the course? What would be the course were 